Modern Physics Letters B

Vol. 31, No. 32 (2017) 1850038 (15 pages)

(C) The Author(s)

DOI: $10.1142 /$ S0217984918500380

\title{
Coherent structures induced by dielectric barrier discharge plasma actuator
}

\author{
Xin Zhang* and Huaxing $\mathrm{Li}^{\dagger}$ \\ School of Aeronautics, \\ Northwest Polytechnical University, \\ Xi'an 710072, China \\ *lookzx@mail.ustc.edu.cn \\ †hxli@nwpu.edu.cn \\ Kwing So Choi ${ }^{\ddagger}$ and Longfei Song $\S$ \\ Faculty of Engineering, University of Nottingham, \\ Nottingham, NG7 2RD, UK \\ $\ddagger$ Kwing-So.Choi@nottingham.ac.uk \\ ${ }^{\S}$ Longfei.Song@nottingham.ac.uk
}

Received 25 July 2017

Revised 29 September 2017

Accepted 1 November 2017

Published 17 November 2017

\begin{abstract}
The structures of a flow field induced by a plasma actuator were investigated experimentally in quiescent air using high-speed Particle Image Velocimetry (PIV) technology. The motivation behind was to figure out the flow control mechanism of the plasma technique. A symmetrical Dielectric Barrier Discharge (DBD) plasma actuator was mounted on the suction side of the SC (2)-0714 supercritical airfoil. The results demonstrated that the plasma jet had some coherent structures in the separated shear layer and these structures were linked to a dominant frequency of $f_{0}=39 \mathrm{~Hz}$ when the peak-to-peak voltage of plasma actuator was $9.8 \mathrm{kV}$. The high speed PIV measurement of the induced airflow suggested that the plasma actuator could excite the flow instabilities which lead to production of the roll-up vortex. Analysis of transient results indicated that the roll-up vortices had the process of formation, movement, merging and breakdown. This could promote the entrainment effect of plasma actuator between the outside airflow and boundary layer flow, which is very important for flow control applications.
\end{abstract}

Keywords: Coherent structures; plasma actuator; flow control; dielectric barrier discharge.

This is an Open Access article published by World Scientific Publishing Company. It is distributed under the terms of the Creative Commons Attribution 4.0 (CC-BY) License. Further distribution of this work is permitted, provided the original work is properly cited. 


\section{Zhang et al.}

\section{Introduction}

Flow control using Dielectric Barrier Discharge (DBD) plasma actuator is a booming field of investigation. Thanks to its simple construction, convenient installation and fast time response, the plasma actuator has attracted much attention in aeronautical applications. ${ }^{1-13}$ Typical asymmetrical DBD plasma actuators include an exposed and covered electrode separated by a thin insulating material, with the covered electrode encapsulated by the dielectric material and the exposed electrode exposed to the air, as shown in Fig. 1. When the two electrodes are connected to a high-voltage, high-frequency AC power supply, the air around the exposed electrode is ionized, producing a body force that acts on the surrounding air. This induces a wall jet on the edge of the exposed electrode. As a flow control method, the plasma actuators have been studied on various aerodynamic research objects in wind tunnel, such as airfoil,,$\underline{14-16}$ wing, $, \frac{17-20}{-2 y l i n d e r, ~}, 21-24$ turbines blade,, 25 and compressor cascade. ${ }^{26,27}$

Apart from the wind tunnel experiments of flow control, many researchers have focused on the induced flow field by DBD plasma actuators in quiescent air. ${ }^{28}-\underline{32}$ Characterizing the induced airflow is a priority to understand the mechanism of plasma flow control. The velocity of plasma jet was first measured in 1998. As a pioneer of this research field, Roth et al. measured the jet velocity downstream of the exposed electrode using a Pitot tube for the first time in this study. $\frac{33}{}$ After that, lots of researchers devote themselves to be engaged in these investigations. Johnson and Scott obtained the jet velocity profiles at several locations downstream of plasma actuator by hot wire. $\frac{34}{4}$ Forte et al. $\frac{35}{5}$ and Enloe et al. $\frac{36}{}$ carried out some investigations into the velocity of plasma jet in order to find the optimum parameters, such as geometrical, materials and electrical parameters. Corke et al. showed the induced airflow field using time-averaged PIV. .37 Jukes and Choi found the velocity profile of plasma jet was similar to the classical laminar wall jet. $\frac{38}{}$

Some researchers believe that the control effect of plasma actuator depend on the speed of plasma jet. Thus, increasing the velocity of induced jet becomes the

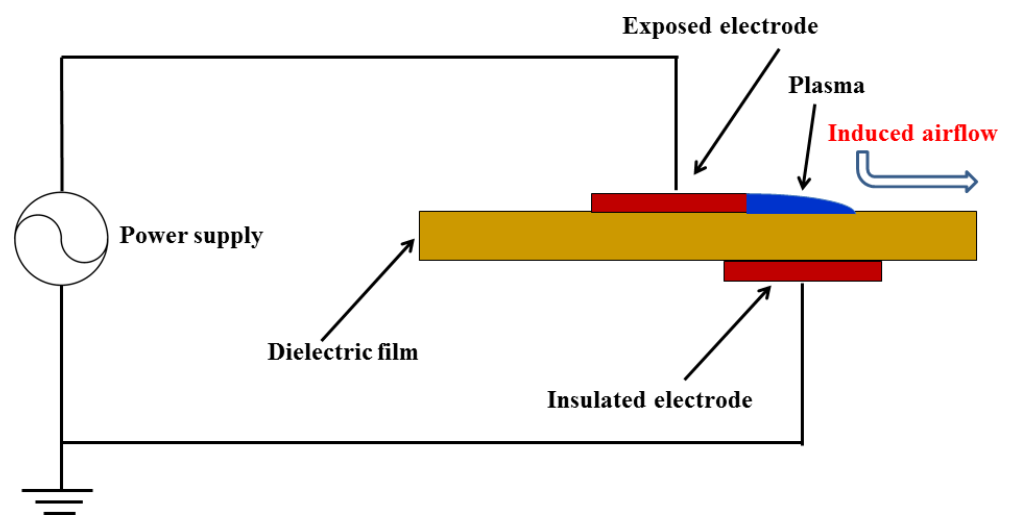

Fig. 1. Typical configuration of DBD plasma actuator. 
main target of investigation in this field. However, the maximum velocity of induced jet by single plasma actuator is no more than $10 \mathrm{~m} / \mathrm{s} \cdot \frac{35}{}$ Compared to the realistic condition, such as taking off, landing and cruising of aircraft, the momentum provided by induced jet is very little. Therefore, it is difficult to control the natural flow at high wind speed or high Reynolds number with such very limited momentum input. Nevertheless, some researches have shown the flow separation around the wing which can be suppressed by AC plasma actuator at the incoming speed of $130 \mathrm{~m} / \mathrm{s}^{19}$ and at Reynolds number $2 \times 10^{6} . \underline{39}$ These results indicated that the stable plasma jet cannot comprehensively describe the induced airflow by plasma actuator. There is some unknown phenomenon of induced airflow by plasma actuator that we should explore and investigate. Thus, the speed of the plasma wall jet is not the only criterion for judging the control ability of plasma actuator. The effect of the flow instabilities in the separated shear layer of induced airflow, as well as the transition process, such as fundamental frequency of separated shear layer, could need an in-depth study.

The main goal of this paper is to study the development process of coherent structures in the shear layer induced by plasma actuator. Here, the airflow induced by plasma actuator was experimentally studied in a closed chamber using high-speed PIV technology. In order to be close to the wind tunnel experiments, a supercritical airfoil with a symmetrical DBD plasma actuator which has a better control effect at high speed wind and high Reynolds number comparing to the traditional asymmetrical plasma actuator $\frac{16,39}{}$ was used. The results illustrated that the evolution process of roll-up vortices and shed light on the relationship between the frequencies in the power spectra and the coherent structures.

\section{Experimental Setup}

The LAVISION PIV equipment was used to obtain the transient behavior of flow field induced by plasma actuator. Figure 2 shows the equipment layout of the PIV experiments. The testing model with plasma actuator was placed in a square chamber with the size of $600 \mathrm{~mm}$ (width) $\times 600 \mathrm{~mm}$ (height) $\times 800 \mathrm{~mm}$ (length) to make sure that the induced flow field was not affected by the environment. The PIV laser was mounted on top of the chamber and the high-speed CCD camera which had a spatial resolution of 1024 pixels $\times 1024$ pixels was installed on the side. The area of view field was $18 \mathrm{~mm} \times 18 \mathrm{~mm}$ and image pairs were recorded at a frequency of $3000 \mathrm{~Hz}$. The sampling duration was 6 s. The DEHS (Di-ethyl-hexyl-sebacate) particles which were used to seed the air through the input of chamber were about $1 \mu \mathrm{m}$ diameter and generated by LASKIN-40 smoker. The velocity and vorticity fields of induced airflow were calculated by LAVISION PIV software, using a cross-correlation algorithm to generate vectors over a $16 \times 16$ pixel interrogation window with $50 \%$ overlap. The five small holes which were in the lower left corner of chamber were used for cable running. 


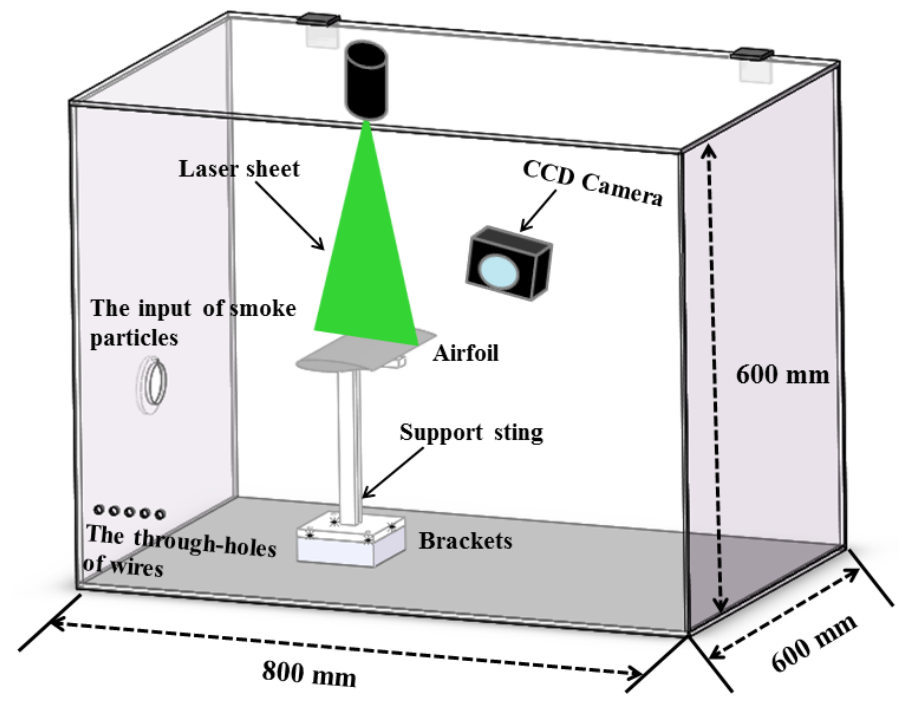

Fig. 2. Schematic of the experimental set-up.

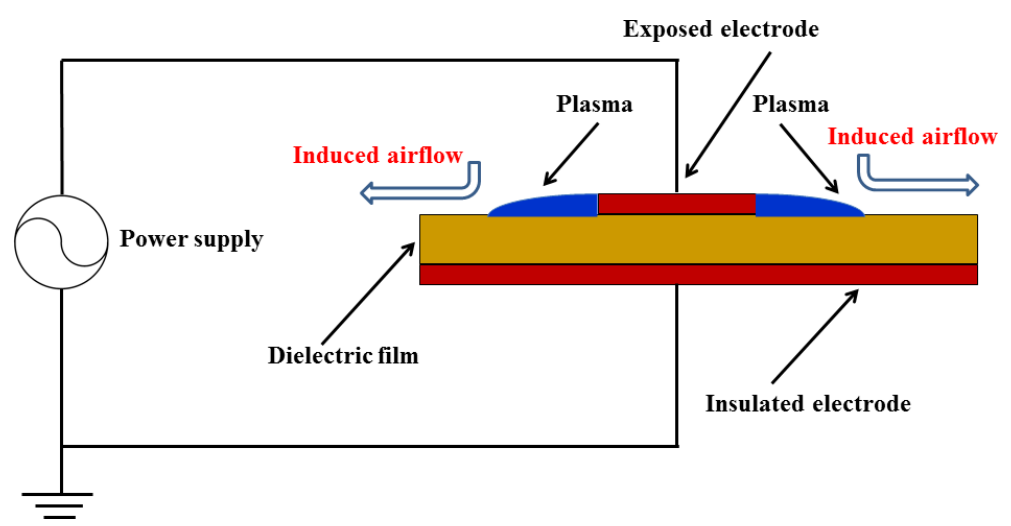

Fig. 3. Electrode configuration of a symmetrical DBD plasma actuator.

A symmetrical plasma actuator was adopted in this study. The main layout difference between an asymmetrical plasma actuator and a symmetrical one is the lower electrode, as shown in Fig. 3. The lower electrode of the symmetrical plasma actuator covers the whole upper electrode, thus this actuator could generate bidirectional plasma on both sides of the upper electrode.

Figure 4 shows the schematic diagram of airfoil and a symmetrical plasma actuator. In this work, the exposed electrode which was mounted on the suction side of the SC (2)-0714 supercritical airfoil near the leading edge was copper foil tape. It was $2 \mathrm{~mm}$ wide and $0.02 \mathrm{~mm}$ thick. The airfoil had a chord length $c$ of $36 \mathrm{~mm}$ and a spanwise length of $240 \mathrm{~mm}$. The covered electrode was the whole testing model which was made of aluminum. The insulating film was kapton which possessed 


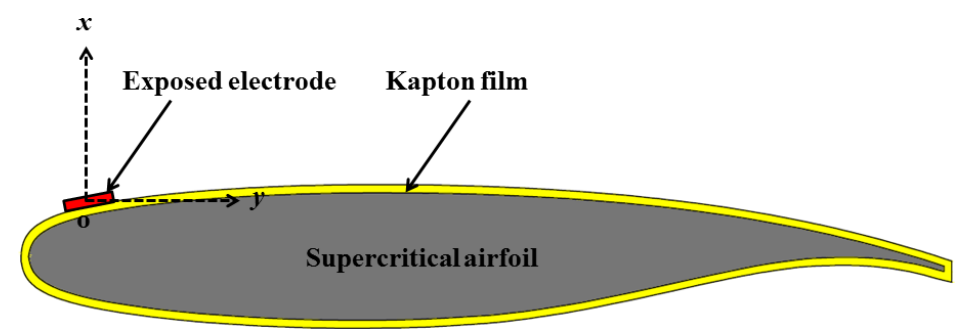

Fig. 4. Scheme of electrode configuration of the symmetrical plasma actuator.

the outstanding thermal, mechanical, chemical and electrical properties and had been widely used in the study of plasma flow control. The origin of the coordinate system was fixed at the midpoint of exposed electrode. The $X$-axis paralleled to the streamwise flow and the $Y$-axis pointed the vertical direction.

\section{Results and Discussion}

\subsection{Time-averaged characteristics}

Figure 5 presents the time-averaged velocity fields above the upper electrode in quiescent air. Here, $f$ is the frequency of the power source, $U_{p-p}$ is the peak-to-peak voltage amplitude, $x^{*}(=x / c)$ is the non-dimensional distance in the horizontal direction and $y^{*}(=y / c)$ is the non-dimensional distance in the normal direction. The electrical and geometrical parameters were fixed in subsequent experiments. The surrounding air is deflected towards the actuator, and then ejects in the vicinity of the airfoil surface from the upper electrode. The induced airflow is similar to a traditional wall jet. But there is no mass added to the airflow. $\frac{40}{}$ Due to the

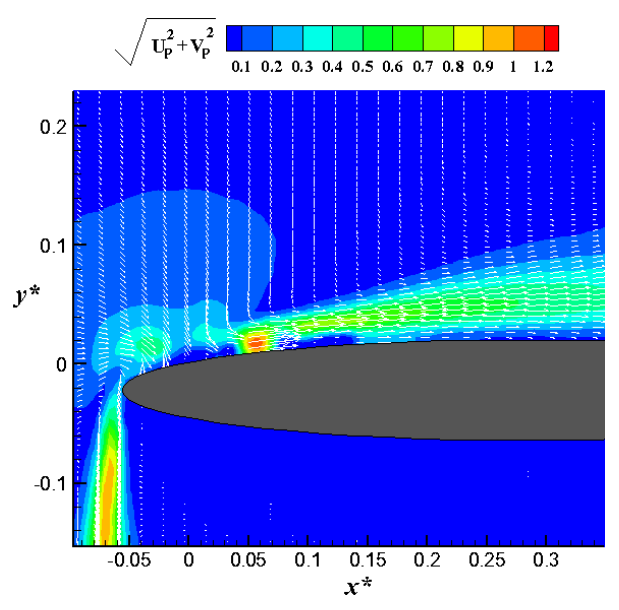

(a) $\mathrm{U}_{\mathrm{p}-\mathrm{p}}=6.4 \mathrm{kV}, \mathrm{f}=3 \mathrm{kHz}$

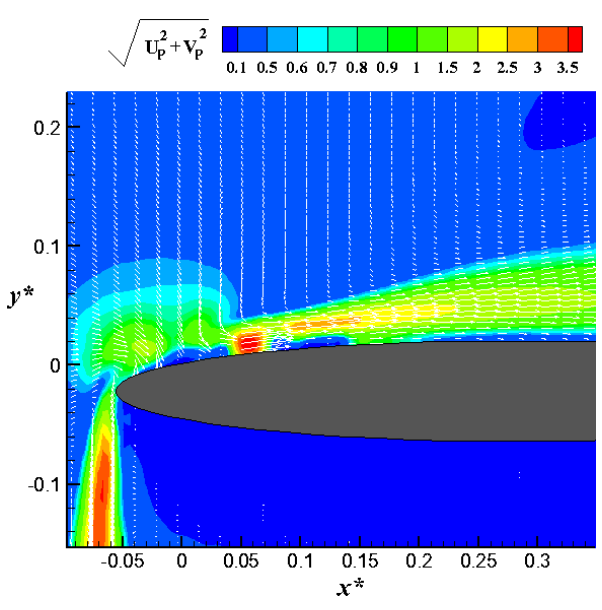

(b) $\mathrm{U}_{\mathrm{p}-\mathrm{p}}=9.8 \mathrm{kV}, \mathrm{f}=3 \mathrm{kHz}$

Fig. 5. (Color online) Time-averaged velocity field above the actuator in quiescent air. 


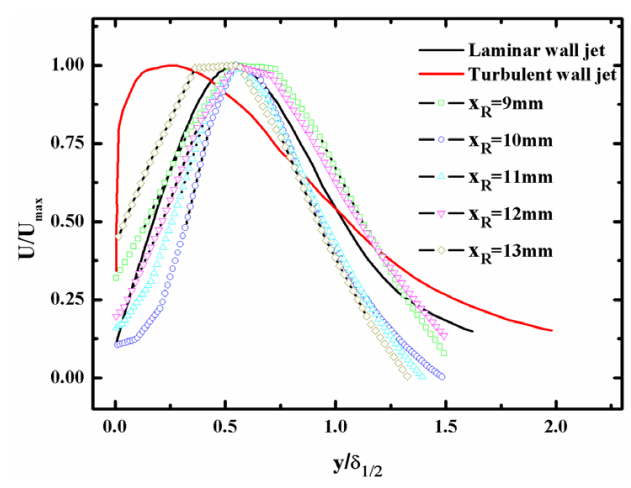

(a) $\mathrm{U}_{\mathrm{p}-\mathrm{p}}=6.4 \mathrm{kV}, \mathrm{f}=3 \mathrm{kHz}$

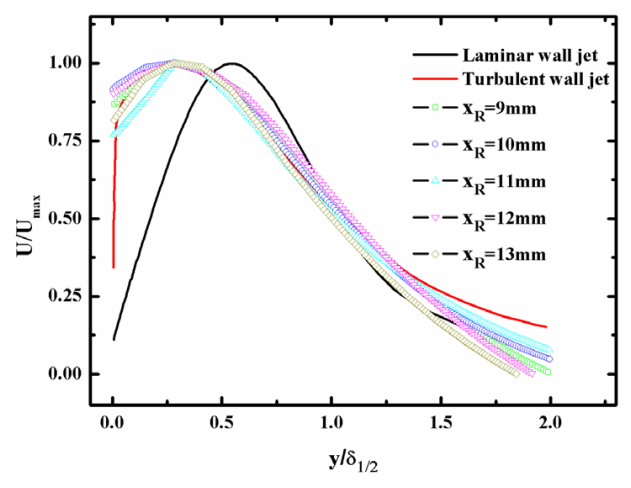

(b) $\mathrm{U}_{\mathrm{p}-\mathrm{p}}=9.8 \mathrm{kV}, \mathrm{f}=3 \mathrm{kHz}$

Fig. 6. (Color online) Non-dimensional velocity profiles of the DBD plasma wall jet.

symmetrical actuator, the actuator induces a bi-directional wall jet on both sides of the upper electrode. The maximum velocities of two jets are approximately equal. In addition, the maximum velocity of plasma jet increases with the voltage amplitude.

Figure 6 shows the non-dimensional velocity profile of plasma wall jet over the airfoil upper surface at the different voltage amplitudes. Here, $U_{\max }$ is the local maximum velocity of jet, $\delta_{1 / 2}$ is the wall normal position at which the speed of jet has decreased to $0.5 U_{\max }$. The velocity profile of plasma wall jet is quite similar to the velocity profile of laminar wall jet at $U_{p-p}=6.4 \mathrm{kV}$. These agree with Juke's results. ${ }^{38}$ However, at $U_{p-p}=9.8 \mathrm{kV}$, the non-dimensional velocity profile is well consistent with the classic velocity profile of turbulent wall jet. Therefore, laminar wall jet could not furnish a comprehensive description of the plasma wall jet. The voltage amplitudes influence the characterization of plasma wall jet. These findings could change the traditional conception in plasma jet and will be proved in this section using other data, such as the Reynolds number of plasma wall jet and transient flow field structures.

The Reynolds number of plasma wall jet over the airfoil upper surface is shown in Fig. 7. $\operatorname{Re}_{J}\left(\operatorname{Re}_{J}=U_{\max } \delta_{1 / 2} / \nu\right)$ represents the Reynolds number of jet. Compared with the plasma actuator under high voltage condition, the Reynolds number is relatively low and the maximum is no more than 40 . However, at $U_{p-p}=9.8 \mathrm{kV}$, the Reynolds number increases over the surface and the value is higher than in the case of lower voltage amplitude. According to the previous results, the critical Reynolds number of a laminar wall-jet is 57.41 The plasma wall jet could start to experience the transition process when the Reynolds number of plasma wall jet has exceeded the critical value. These results suggested that the higher voltage amplitude may trigger the transition of induced jet. In addition, the Reynolds number of plasma jet is one of the important criteria to judge the characterization of plasma wall jet. 


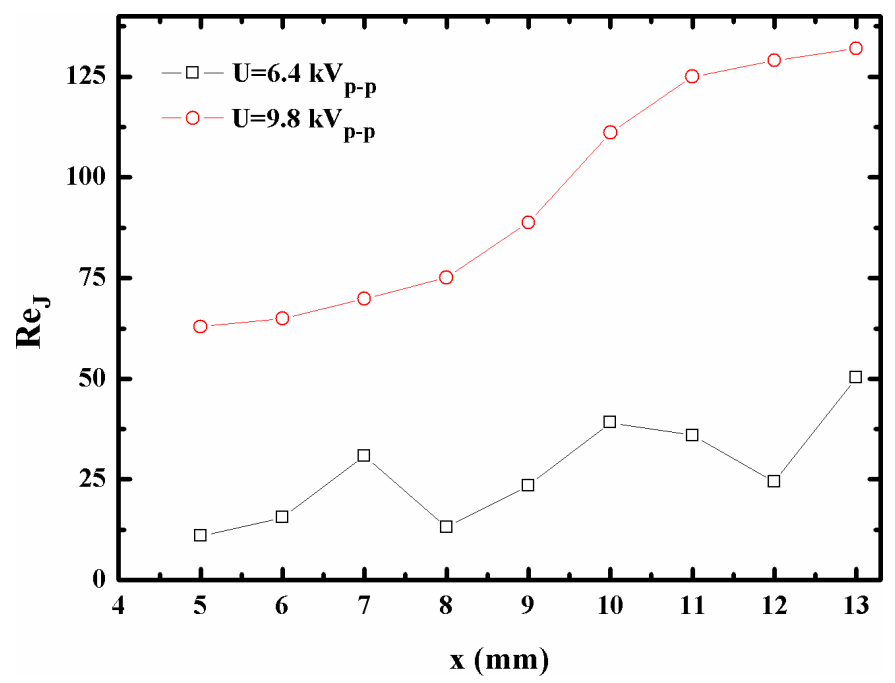

Fig. 7. Reynolds number of plasma jet over the surface in quiescent air.

\subsection{Transient flow field structure}

Figure 8 is a snapshot of the flow field induced by plasma actuator in quiescent air. Since the plasma actuator is activated, the mutual effect between the plasma and the surrounding air leads to a starting vortex which locates above the exposed electrode. There are no coherent structures near the wall when the peak-to-peak voltage amplitude is $6.4 \mathrm{kV}$, as presented in Fig. 8(a). However, as the voltage amplitude is enhanced, the induced airflow has some coherent structures, such as roll-up vortex and secondary vortex, which are close to the airfoil upper surface, as depicted in Fig. 8(b). When the Reynolds number of plasma jet is beyond the critical value, the shear layer of plasma wall jet becomes unstable. The induced airflow generates a roll-up vortex in the shear lay of plasma wall jet. As time goes on, the roll-up vortices stretch and move along the airfoil upper surface and form a train of vortices. Meanwhile, in order to preserve the no-slip boundary condition,

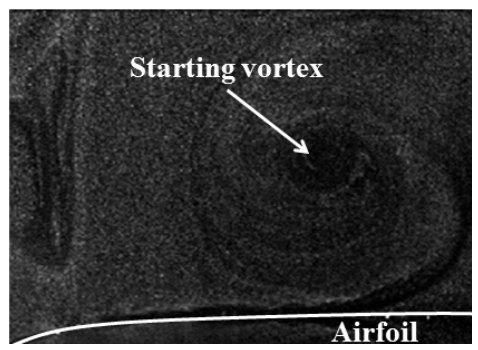

(a) $\mathrm{U}_{\mathrm{p}-\mathrm{p}}=6.4 \mathrm{kV}, \mathrm{f}=3 \mathrm{kHz}$

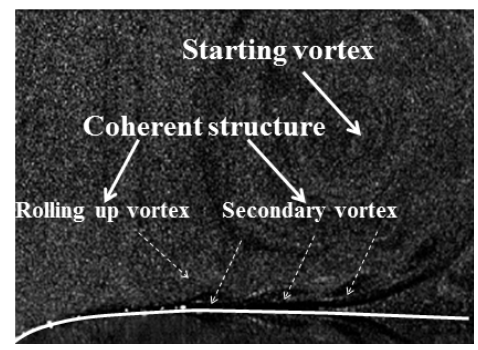

(b) $\mathrm{U}_{\mathrm{p}-\mathrm{p}}=9.8 \mathrm{kV}, \mathrm{f}=3 \mathrm{kHz}$

Fig. 8. Original PIV images of the flow field induced by plasma actuator in quiescent air $(t=0.85 \mathrm{~s})$. 


\section{Zhang et al.}

secondary vortices are formed over the airfoil upper surface. The secondary vortices are underneath the roll-up vortices and move along the airfoil surface.

One important thing to note is that the disparity between the roll-up vortex and the starting vortex. Initially, the formation mechanism is different. When the plasma actuator reaches the threshold voltage, the air near the upper electrode could be ionized and carry the momentum to the flow field. To replenish the flow that has been ejected, entrainment occurs above the upper electrode to generate a starting vortex. However, the roll-up vortices could be generated due to the instability of shear layer. In addition, the size of starting vortex is bigger than roll-up vortex's size. Meanwhile, the locations of the vortex cores are different between the starting vortex and the roll-up vortices. The starting vortex could roll-up and move away from the wall. The moving trajectory of roll-up vortex, however, is near the wall. Moreover, the starting vortex is a single vortical structure and roll-up vortices are a train of vortices. The roll-up vortices have the process of formation, movement, merging and breakdown.

To give the quantitative comparison of these two kinds of cases, swirling strength $\left(\Lambda_{\mathrm{ci}}\right)$ field induced by the plasma actuator is shown in Fig. 9 . Here, $\Lambda_{\mathrm{ci}}$ is defined by $\Lambda_{\mathrm{ci}} \equiv \lambda_{\mathrm{ci}} \frac{\omega}{|\omega|}$, where $\lambda_{\mathrm{ci}}$ is the imaginary part of the complex eigenvalue of the velocity gradient tensor, ${ }^{42}$ which can represent the vortical structure, $\omega$ is the vorticity. The sign of local vorticity is assigned to $\lambda_{\text {ci }}$ for identifying the sense of rotation. $\stackrel{43}{ }$

The swirling strength mainly concentrates around the starting vortex and the edge of the exposed electrode at $U_{p-p}=6.4 \mathrm{kV}$, as presented in Fig. 9(a). There is no significant swirling strength near the wall. However, when the voltage amplitude is improved, the swirling strength near the airfoil upper surface as well as around the starting vortex is visible, as shown in Fig. 9(b). The negative swirling strength which is attached to the wall surface represents the secondary vortices. Some discrete areas above the secondary vortices are roll-up vortices which are positive.

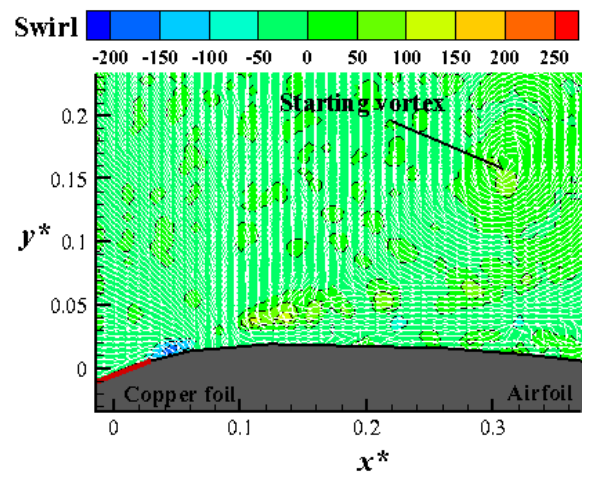

(a) $\mathrm{U}_{\mathrm{p}-\mathrm{p}}=6.4 \mathrm{kV}, \mathrm{f}=3 \mathrm{kHz}$

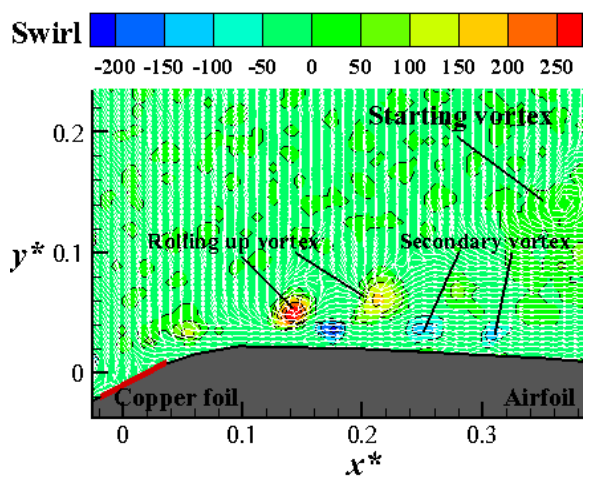

(b) $\mathrm{U}_{\mathrm{p}-\mathrm{p}}=9.8 \mathrm{kV}, \mathrm{f}=3 \mathrm{kHz}$

Fig. 9. (Color online) Swirling strength field induced by the DBD plasma actuator in quiescent air. 


\subsection{Coherent structures}

From the discussion above, the results suggested that the characterization of plasma jet is related to the voltage amplitude. When the voltage amplitude is higher, the plasma jet could be the turbulent jet which includes coherent structures near the wall. These could promote the mixing effect of plasma actuator which is beneficial for the separation flow control at high wind speed and high Reynolds number. Therefore, the coherent structures induced by plasma actuator will be analyzed in this section.

According to the transient flow field results, the roll-up vortices and secondary vortices move to the trailing edge of airfoil. Although the induced flow field is unsteady, it still has quasi-periodic characteristics, as indicated by the power spectrum of velocity data in the shear layer over the surface shown in Fig. 11. The locations of the calculation point $A$ to $C$ are given in Fig. 10. In order to get the precise comparison of the power spectrum, the calculation points are collected at a $y$ position that corresponds to the maximum r.m.s velocity.

Figure 11 shows power spectra of the vertical fluctuating velocity at different places of airfoil with different voltage amplitudes. It is evident from Fig. 11 that there is no distinct dominant frequency at three positions when the peak-to-peak

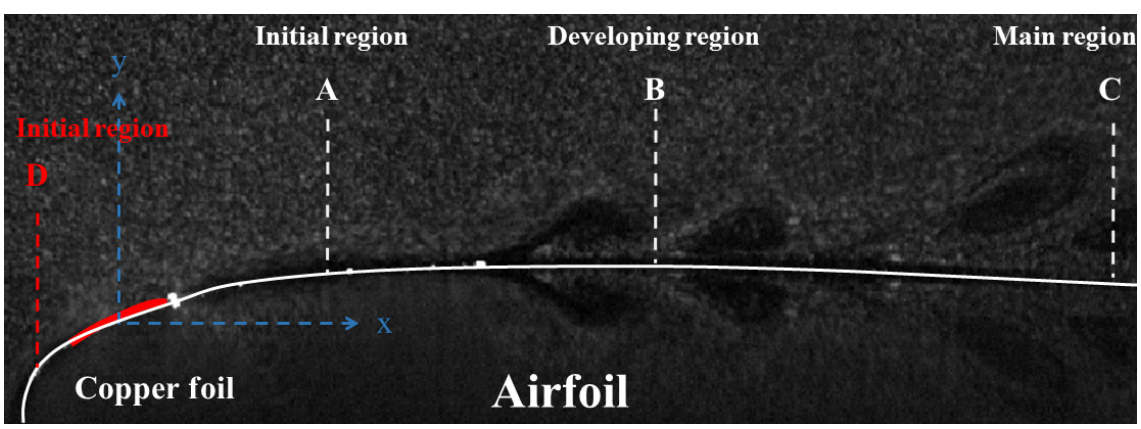

Fig. 10. The locations of the calculation point $A$ to $C$ on the right side of the exposed electrode $\left(x_{A}=3.2 \mathrm{~mm} ; x_{B}=6.5 \mathrm{~mm} ; x_{C}=13.3 \mathrm{~mm}\right)$.

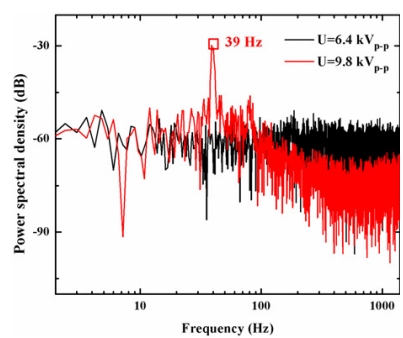

(a) Point A

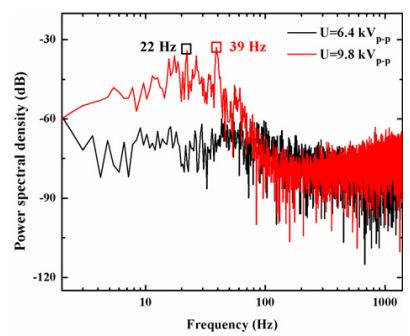

(b) Point B

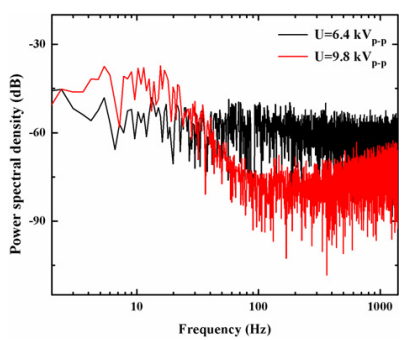

(c) Point C

Fig. 11. (Color online) Power spectra of the vertical fluctuating velocity at different locations of the right side of the exposed electrode. 


\section{Zhang et al.}

voltage amplitude is $6.4 \mathrm{kV}$. However, at point $A$, a dominant frequency of $f_{0}=$ $39 \mathrm{~Hz}$ is amplified in the shear layer when the plasma actuator is excited by higher voltage. Due to the instability of shear layer, it could force the vortex to roll-up in the shear layer. Then the disturbances start to grow and a sub-harmonic of the dominant frequency of $22 \mathrm{~Hz}$ is emerged at point $B$, as shown in Fig. 11(b). It suggested that the roll-up vortices could be merged and the interactions between the disturbances. ${ }^{44}$ However, note that the velocity spectrum in Fig. 11(b) displays a peak at $22 \mathrm{~Hz}$ which is somewhat less pronounced than a fundamental peak at $39 \mathrm{~Hz}$. That means that the merging of the roll-up vortices in the separated shear layer could not be very notable. Further downstream, there is no distinct peak in the power spectra, as depicted in Fig. 11(c). It indicated that the roll-up vortices rapidly broke down during the transition process.

According to the fundamental frequency $f_{0}$ of $39 \mathrm{~Hz}$, the period was obtained. Figure 12 shows a periodic formation of the coherent structures in the induced flow

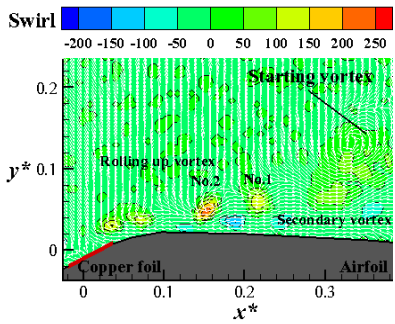

(a) $\mathrm{t}=0$

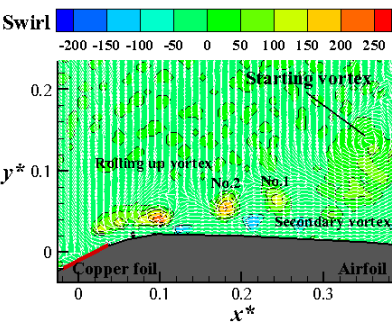

(d) $\mathrm{t}=3 / 8 \mathrm{~T}$

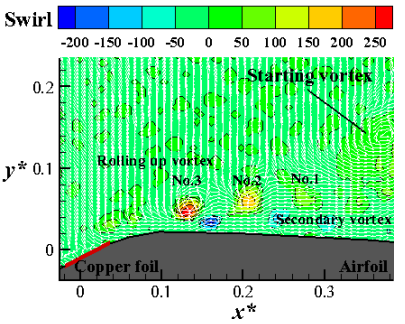

(g) $\mathrm{t}=6 / 8 \mathrm{~T}$

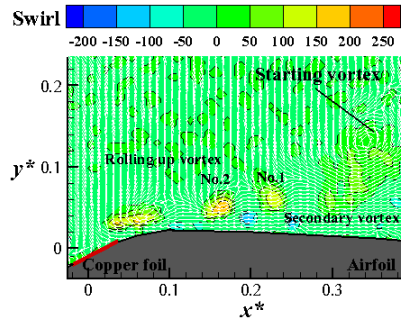

(b) $\mathrm{t}=1 / 8 \mathrm{~T}$

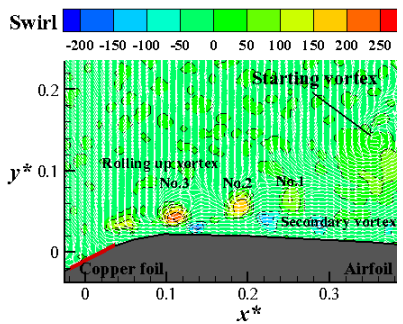

(e) $\mathrm{t}=4 / 8 \mathrm{~T}$

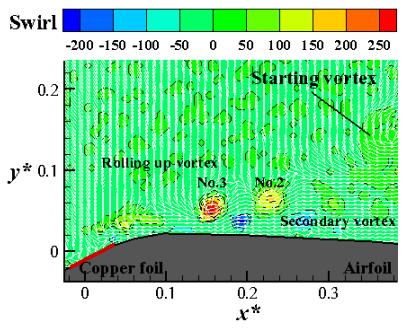

(h) $\mathrm{t}=7 / 8 \mathrm{~T}$

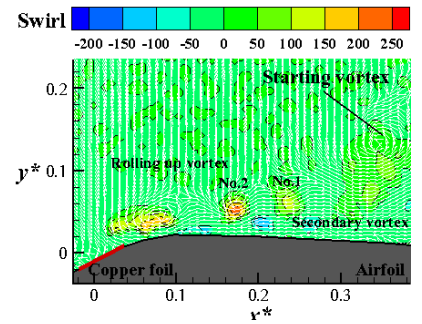

(c) $\mathrm{t}=2 / 8 \mathrm{~T}$

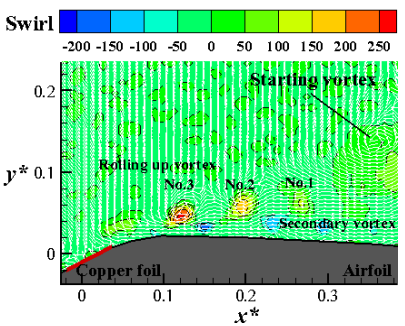

(f) $\mathrm{t}=5 / 8 \mathrm{~T}$

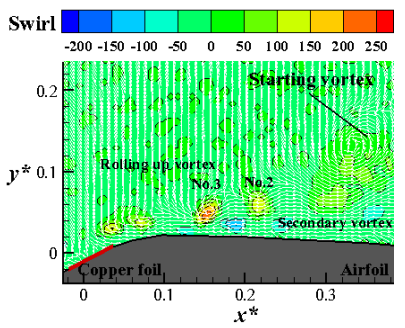

(i) $\mathrm{t}=\mathrm{T}$

Fig. 12. (Color online) Evolution of swirling strength field on the right side of the exposed electrode in one cycle ( $T$ stands for cycle time). 
field. Based on the swirling strength with velocity vector in these pictures, we can see there are several vortices in the flow field.

First, the two single roll-up vortical structures which are marked as No. 1 and No. 2 are generated in the separated shear layer. The two roll-up vortices move together toward the downstream. Furthermore, the spacing of the two roll-up vortices

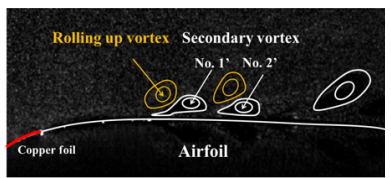

(a) $\mathrm{t}=0$

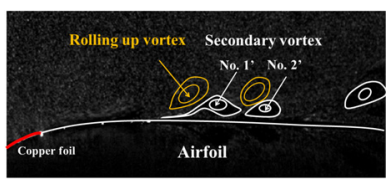

(d) $\mathrm{t}=3 / 8 \mathrm{~T}$

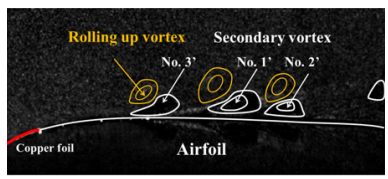

(g) $\mathrm{t}=6 / 8 \mathrm{~T}$

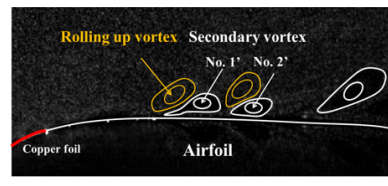

(b) $\mathrm{t}=1 / 8 \mathrm{~T}$

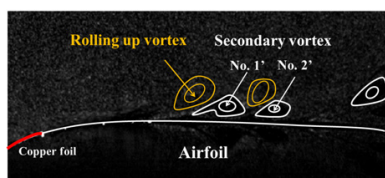

(e) $\mathrm{t}=4 / 8 \mathrm{~T}$

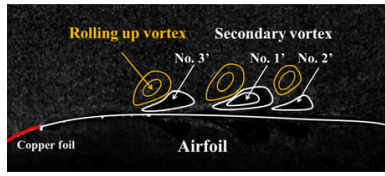

(h) $\mathrm{t}=7 / 8 \mathrm{~T}$

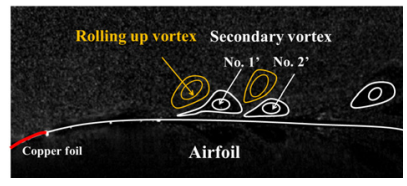

(c) $\mathrm{t}=2 / 8 \mathrm{~T}$

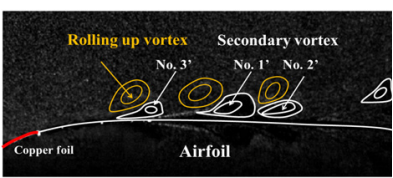

(f) $\mathrm{t}=5 / 8 \mathrm{~T}$

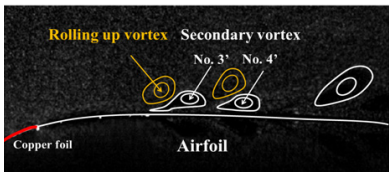

(i) $\mathrm{t}=\mathrm{T}$

Fig. 13. (Color online) Evolution process of the secondary vortices on the right side of the exposed electrode in one cycle.

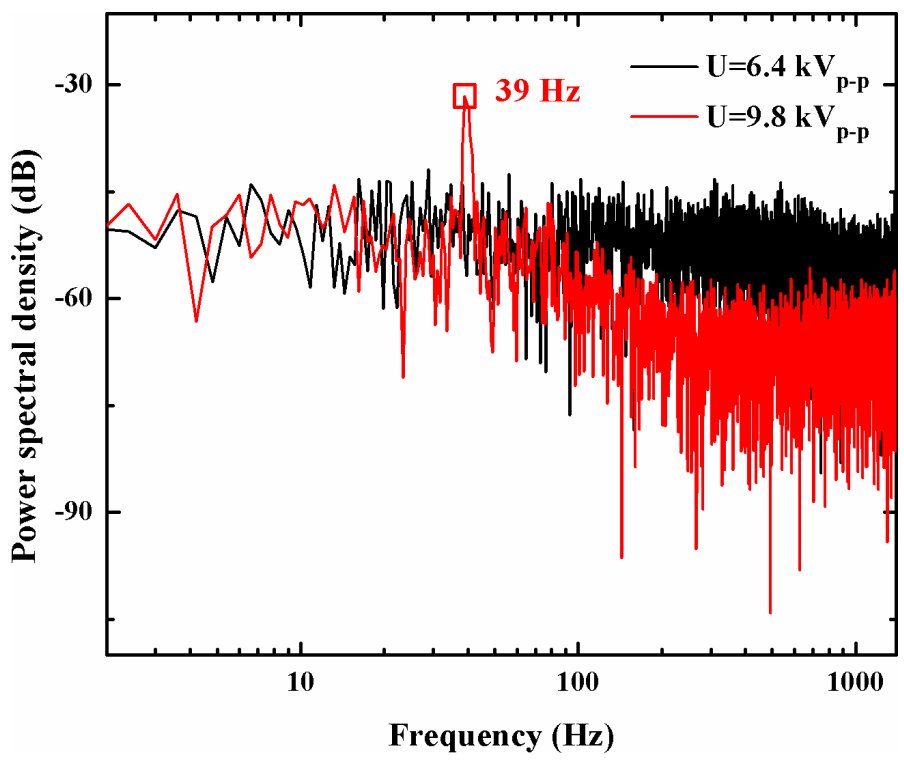

Fig. 14. (Color online) Power spectra of the vertical fluctuating velocity at the initial region of the left side of the exposed electrode. 


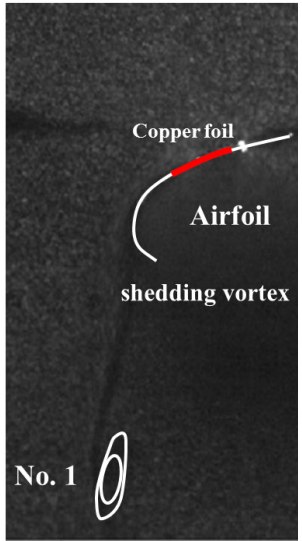

(a) $\mathrm{t}=0$

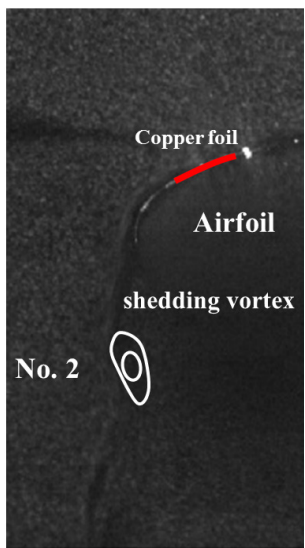

(d) $\mathrm{t}=3 / 8 \mathrm{~T}$

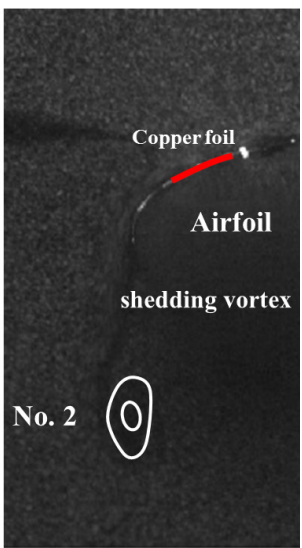

(g) $t=6 / 8 \mathrm{~T}$

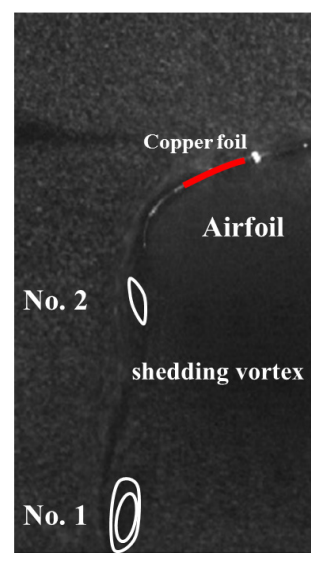

(b) $t=1 / 8 \mathrm{~T}$

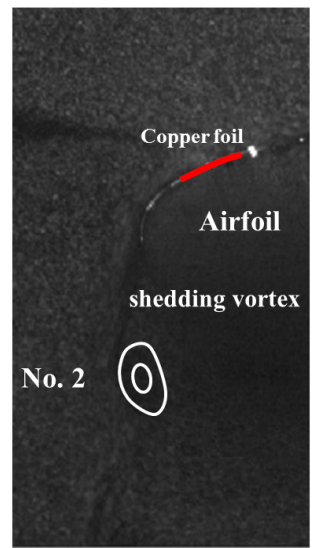

(e) $\mathrm{t}=4 / 8 \mathrm{~T}$

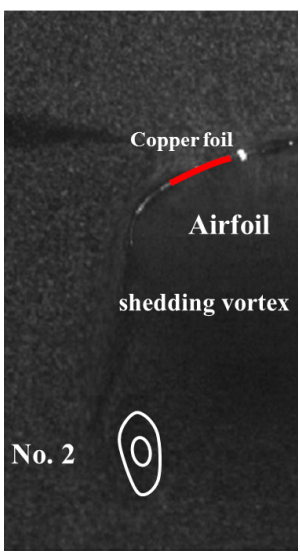

(h) $\mathrm{t}=7 / 8 \mathrm{~T}$

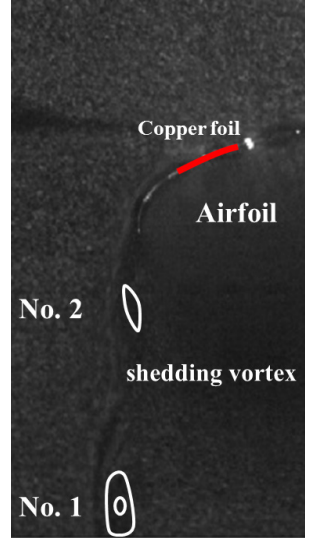

(c) $\mathrm{t}=2 / 8 \mathrm{~T}$

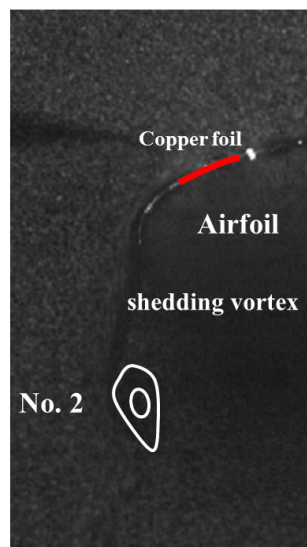

(f) $\mathrm{t}=5 / 8 \mathrm{~T}$

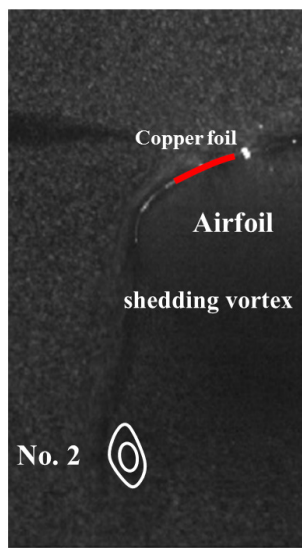

(i) $\mathrm{t}=\mathrm{T}$

Fig. 15. (Color online) Evolution process of the roll-up vortices on the left side of the exposed electrode in one cycle. 
is approximately constant in these images, suggesting that they are shed at constant frequency. Then another roll-up vortex which is labeled as No. 3 is induced, as shown in Fig. 12(e). As time goes by, the breakdown of No. 1 vortex occurs at the downstream of airfoil, as depicted in Fig. 12(h). After that, the two vortices, namely No. 2 and No. 3, move to the trailing edge of airfoil, which means that the roll-up vortexes experience one period, as shown in Fig. 12(i).

Not only the roll-up vortices but also the secondary vortices showed quasiperiodic characteristics. Figure 13 shows the original PIV image in one cycle. These pictures describe the change process of the secondary vortices, such as formation, roll-up, movement and break down of vortices.

In addition, the roll-up vortices on the left side of the exposed electrode also are analyzed in this part. The location of the calculation point $D$ is at $x=-2.7 \mathrm{~mm}$, $y=-4.6 \mathrm{~mm}$, which is depicted in Fig. 10 .

Figure 14 shows power spectra of the vertical fluctuating velocity at point $D$ for different voltage amplitude. It is noteworthy that there is no distinct dominant frequency like other three positions when the peak-to-peak voltage amplitude is $6.4 \mathrm{kV}$. However, there is a primary frequency of $f_{0}=39 \mathrm{~Hz}$ when the plasma actuator is driven by higher voltage. The results indicated that the fundamental frequency $f_{0}$ of roll-up vortices on both sides of exposed electrode is equal. It implied that the fundamental frequency related to the electric parameters, such as the frequency of power supply, but is barely relevant to the location of plasma actuator.

Figure 15 shows the changing process of the roll-up vortices on the left side of the symmetrical plasma actuator in one period. The results indicated that the evolution of roll-up vortices also has quasiperiodic characteristics and just like vortexshedding phenomenon due to the Coanda effect.

\section{Conclusions and Discussion}

The flow field induced by a symmetrical DBD plasma actuator which is mounted on the suction side of the SC (2)-0714 supercritical in quiescent air has been investigated using high-speed PIV technology. The results suggested that the plasma jet could generate two types of jet, laminar jet and turbulent jet, which can be influenced by the voltage amplitudes. When the voltage amplitude is low, the plasma jet is laminar jet. The plasma jet becomes the turbulent jet as the voltage amplitude is increased. The Reynolds number is one of valid criteria to evaluate the characterization of plasma wall jet.

In addition, thanks to the high voltage amplitude, the turbulent jet induced by plasma actuator contains some coherent structures, such as roll-up vortices and secondary vortices. There are some distinct differences between these vortical structures and starting vortex, such as the formation mechanism, the movement trajectory, and the size. Meanwhile, these coherent structures are a train of vortices which have the process of formation, movement, merging and breakdown and the starting 


\section{$X$. Zhang et al.}

vortex is a single vortical structure. What is more, spectral analysis of the vertical fluctuating velocity indicated that there is a fundamental frequency of $f_{0}=39 \mathrm{~Hz}$ in the shear layer when the plasma actuator is driven by the high voltage amplitude. Then the peak in the power spectra could disappear due to the breakdown of vortices. Moreover, because of the symmetrical configuration of plasma actuator, the two wall jets which are flowing on both sides of the exposed electrode have the same dominant frequency.

Based on the above, the laminar jet cannot comprehensively describe the characterization of plasma jet. When the plasma actuator induces the laminar jet, the velocity of jet could mainly affect the control effect. The momentum input is the major controlling mechanism. While the voltage amplitude is increased, the plasma jet becomes the turbulent jet which contains some coherent structures and much energy in the vicinity of wall surface. These structures could enhance the entrainment effect of plasma actuator between the outside airflow and boundary layer flow through rolling and moving. Therefore, the plasma actuator which is driven by high voltage amplitude has two controlling mechanisms for separation flow control, mixing and momentum input, which are very vital to flow control at high wind speed and high Reynolds number. Further study will figure out the relationship between the dominant frequency and actuation parameters, such as the voltage amplitude and frequency of power supply.

\section{Acknowledgments}

This work is supported by the fund of equipment investigation in advance "Flow control on a wing using plasma actuator and unsteady blowing and suction" (Project No. 51313010204).

\section{References}

1. T. C. Corke, M. L. Post and D. M. Orlov, Exp. Fluids 46 (1987) 1.

2. Z. J. Zhao, C. Gao, F. Liu and S. J. Luo, Mod. Phys. Lett. B 24 (2010) 1405.

3. Y. H. Li, Y. Wu and J. Li, Int. J. Flow Control 4 (2012) 1.

4. J. N. Wang, H. X. Li, F. Liu and S. J. Luo, Mod. Phys. Lett. B 24 (2010) 1401.

5. J. J. Wang, K. S. Choi, L. H. Feng, T. N. Jukes and R. D. Whalley, Prog. Aerosp. Sci. 5 (2013) 1.

6. X. K. Che, T. Shao, W. S. Nie and P. Yan, J. Phys. D: Appl. Phys. 45 (2012) 1.

7. Y. Wu, Y. H. Li and M. Jia, Chinese J. Aeronaut. 23 (2010) 39.

8. X. S. Meng, Z. X. Guo and S. J. Luo, Acta Aeronautica et Astronautica Sinica 3 (2010) 500 .

9. P. F. Zhang, A. B. Liu and J. J. Wang, Sci. China Technol. Sci. 53 (2010) 2772.

10. Y. Li, X. Zhang and X. Huang, Exp. Fluids 49 (2010) 367.

11. C. L. Enloe, T. E. McLaughlin, R. K. VanDyken, K. D. Kachner, E. J. Jumper and T. C. Corke, AIAA J. 42 (2004) 589.

12. D. Roupassov, A. Nikipelov, M. Nudnova and A. Starikovskii, AIAA J. 47 (2009) 168.

13. B. Mertz and T. C. Corke, J. Fluid Mech. 669 (2011) 557. 
14. L. H. Feng, T. N. Jukes and K. S. Choi, Exp. Fluids 52 (2012) 1533.

15. J. Little, M. Nishihara and I. Adamovich, Exp. Fluids 48 (2010) 521.

16. X. Zhang, H. X. Li and Y. Huang, J. Aircraft 54 (2017) 301.

17. G. Y. Zhao, Y. H. Li and H. Liang, Chinese J. Aeronaut. 28 (2015) 368.

18. M. H. Han, J. Li and Z. G. Niu, Chinese J. Aeronaut. 28 (2015) 377.

19. C. L. Kelley, P. O. Bowles, J. Cooney and T. C. Corke, AIAA J. 52 (2014) 1871.

20. Z. Wu, C. W. Wong, L. Wang, Z. Lu, Y. Zhu and Y. Zhou, Exp. Fluids 56 (2015) 1.

21. W. F. Li, J. S. Cai and J. N. Hao, J. Exp. Fluid Mech. 27 (2013) 17.

22. R. Sosa, G. Artana, N. Benard and E. Moreau, Exp. Fluids 51 (2011) 853.

23. Y. Sung, W. Kim, M. G. Mungal and M. A. Cappelli, Exp. Fluids 41 (2006) 479.

24. Q. Sun, B. Q. Cheng and Y. H. Li, Sci. China Technol. Sci. 56 (2013) 795.

25. J. H. Huang, T. C. Corke and F. O. Thomas, AIAA J. 44 (2006) 51.

26. Y. H. Li, Y. Wu and M. Zhou, Exp. Fluids 48 (2010) 1015.

27. G. Li, Y. J. Xu and J. Q. Lin, Sci. China Technol. Sci. 52 (2009) 3715.

28. Z. W. Shi and B. G. Fan, Acta Aeronautica et Astronautica Sinica 32 (2011) 1583.

29. J. R. Roth, D. M. Sherman and S. P. Wilkinson, AIAA J. 38 (2010) 1166.

30. S. P. Wilkinson, AIAA Paper 2003-1023 January 2003.

31. F. O. Thomas, T. C. Corke, M. Iqbal, A. Kozlov and D. Schatzman, AIAA J. 47 (2009) 2169.

32. R. Durscher and S. Roy, Exp. Fluids 53 (2012) 1165.

33. J. R. Roth, D. M. Sherman and S. P. Wilkinson, AIAA Paper 1998-0328 January 1998.

34. G. A. Johnson and S. J. Scott, AIAA Paper 2001-3052 June 2001.

35. M. Forte, J. Jolibois, J. Pons, E. Moreau, G. Touchard and M. Cazalens, Exp. Fluids 43 (2007) 917.

36. C. L. Enloe, T. E. McLaughlin and R. D. Van Dyken, AIAA J. 42 (2004) 595.

37. T. C. Corke, E. J. Jumper, M. L. Post and D. Orlov, AIAA Paper 2002-350 January 2002.

38. T. N. Jukes, K. S. Choi and G. A. Johnson, AIAA Paper 2004-2216 July 2004.

39. X. Zhang, Y. Huang, X. N. Wang, W. B. Wang, K. Tang and H. X. Li, Chinese J. Aeronaut. 29 (2016) 1237.

40. R. D. Whalley and K. S. Choi, J. Fluid Mech. 703 (2013) 192.

41. D. H. Chun and W. H. Schwarz, Phys. Fluids 10 (1967) 5.

42. J. Zhou, R. J. Adrian, S. Balachandar and T. M. Kendall, J. Fluid Mech. 387 (1999) 353.

43. C. Zhang, C. Pan and J. J. Wang, Exp. Fluids 51 (2011) 5.

44. A. V. Dovgal, V. V. Kozlov and A. Michalke, Prog. Aerosp. Sci. 30 (1994) 61. 